\title{
Mesenchymal Progenitors Aging Highlights a miR-196 Switch Targeting HOXB7 as Master Regulator of Proliferation and Osteogenesis
}

\author{
Olivia Candini, ${ }^{a}$ Carlotta Spano, ${ }^{a}$ Alba Murgia, ${ }^{a}$ Giulia Grisendi, ${ }^{a}$ Elena Veronesi, ${ }^{a}$ \\ Maria Serena Piccinno, a Manuela Ferracin, ${ }^{b}$ Massimo Negrini, ${ }^{b}$ Francesca Giacobbi,

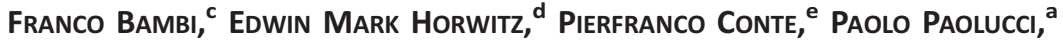 \\ MASSIMO DOMINICI
}

Key Words. Bone marrow stromal cells $•$ Aging $\cdot$ Homeobox genes $•$ Bone

${ }^{\mathrm{a}}$ Department of Medical and Surgical Sciences for Children \& Adults, University-Hospital of Modena and Reggio Emilia, Modena, Italy; ${ }^{\mathrm{b}}$ Department of Morphology, Surgery and Experimental Medicine, Pathology Section and Laboratory for Technologies of Advanced Therapies (LTTA), University of Ferrara, Ferrara, Italy; 'Transfusion Medicine, Children's Hospital A. Meyer, Florence, Italy; ${ }^{d}$ Division of Hematology/Oncology/BMT, Nationwide Children's Hospital, Columbus, Ohio, USA; 'Department of Surgery, Oncology and

Gastroenterology, University of Padova, Istituto Oncologico Veneto IRCCS, Padova, Italy

Correspondence: Massimo Dominici, M.D., Laboratory of Cell Therapies, Department of Medical and Surgical Sciences for Children \& Adults, University Hospital of Modena and Reggio Emilia, Via del Pozzo 71, 41124 Modena, Italy. Telephone: 39059-422-2858; Fax: 39-059-4223341; e-mail: massimo.dominici@unimore.it

Received June 26, 2014; accepted for publication November 1, 2014; first published online in STEM Cells EXPRESS November 26, 2014.

(C) AlphaMed Press 1066-5099/2014/\$30.00/0

http://dx.doi.org/ 10.1002/stem.1897

\section{ABSTRACT}

Human aging is associated with a decrease in tissue functions combined with a decline in stem cells frequency and activity followed by a loss of regenerative capacity. The molecular mechanisms behind this senescence remain largely obscure, precluding targeted approaches to counteract aging. Focusing on mesenchymal stromal/stem cells (MSC) as known adult progenitors, we identified a specific switch in miRNA expression during aging, revealing a miR-196a upregulation which was inversely correlated with MSC proliferation through HOXB7 targeting. A forced HOXB7 expression was associated with an improved cell growth, a reduction of senescence, and an improved osteogenesis linked to a dramatic increase of autocrine basic fibroblast growth factor secretion. These findings, along with the progressive decrease of HOXB7 levels observed during skeletal aging in mice, indicate HOXB7 as a master factor driving progenitors behavior lifetime, providing a better understanding of bone senescence and leading to an optimization of MSC performance. STEM CELLS 2015;33:939-950

\section{INTRODUCTION}

Mesenchymal stromal/stem cells (MSC) have been widely used in preclinical and clinical applications for regenerative medicine mostly relying on their proliferation and differentiation potential [1]. The molecular bases driving these pivotal MSC properties are still under investigation, suggesting the need of a deeper understanding for optimized and enduring benefits. Our group and others observed a transient regenerative potential of mesenchymal progenitors after in vivo infusion, revealing a performance that may be limited by extrinsic and intrinsic factors, as reported for hematopoietic stem cells [2-4]. One of these factors may be represented by an age-related function decline indicating the impairment of key MSC properties during human aging [5-11].

Early observational studies explored several molecular markers of MSC aging, evaluating miRNA and gene expression profile [8, 11-15] or determining the molecular relationship between aging and replicative senescence [9, 16]. However, a proper understanding of agerelated and senescence-related changes remains elusive and further insights are needed to reveal key factors influencing stem cell aging, allowing a deeper knowledge on human senescence, ultimately leading to optimization in regenerative medicine.

Starting from these assumptions, we began to compare adult and child MSC in the attempt to identify molecular features that may be differentially expressed. The initial observation confirmed a significantly higher expression of a marker associated with cell proliferation in children. MicroRNA, as noncoding RNAs involved in gene expression and orchestrating a variety of cellular processes, including aging [17-20], were also considered revealing a small cluster of differentially expressed miRNAs.

We focused on miR-196 being upregulated in aged MSC and having several Homeobox (HOX) genes as targets. HOX are involved in tissue specification and cell identity during embryonic development. In adulthood, HOX proteins are pivotal regulators of either proliferation or differentiation, and compelling evidences suggest a key role for HOX in bone repair $[21,22]$. Among HOX targets our attention was attracted by HOXB7, since its downregulation in aged MSC was preliminarily observed [16]. Thus, we considered the 
interplay between miR-196 and HOXB7 as a potential tool to influence cell behavior overcoming aging interference on MSC properties. Our hypothesis was corroborated by previous findings where an enforced expression of HOXB7 in hematopoietic progenitors stimulates self-renewal, sustaining proliferation, and differentiation [23]. Here, we report that HOXB7 is a specific target of miR-196 in primary bone marrow (BM) MSC, demonstrating that HOXB7 overexpression is associated with a higher proliferative performance and an increase of osteogenic differentiation, opening the way for novel strategies of tissue reconstruction in particular for skeletal disorders.

\section{MATERIALS AND METHOdS}

\section{Patients}

BM harvests were performed from pediatric $(n=7)$ and adult $(n=7)$ patients referring to the Department of Medical and Surgical Sciences for Children \& Adults of the UniversityHospital of Modena after signature of the informed consent. BM harvests were performed for staging in patients with either suspected oncological disease or during major surgical orthopedics intervention. A diagnosis of cancer excluded the samples from the study. Informed consent and procedures were approved by local Ethical Committee.

\section{Animal Studies}

Eleven, ninety, and one hundred and eighty days old male and female $\mathrm{FVB} / \mathrm{n}$ mice (Charles River Laboratory International, Lecco, Italy) were sacrificed and tibia, fibula, and femur were harvested and processed. Six animals were used for each group. Animal protocols were approved by the local Ethical animal Committee.

\section{Histology}

Specimens were fixed with 10\% buffered formalin for 2 days, decalcified in PBS-buffered EDTA (15\%, pH 7.4; Carlo Erba, Milano, Italia) for 14 days, and then paraffin embedded as described [24]. Paraffin sections (5-mm thick) were dehydrated and stained with rabbit anti-human HOXB7 (1:75; Abnova, Walnut, CA) and rabbit anti-human HOXB9 (1:75; Abnova) using a goat anti-rabbit biotinylated secondary $A b$ (1:200; Vector Laboratories, Burlingame, CA) and an avidinbiotin-horseradish peroxidase detection system (Vector Laboratories). Antigen retrieval relied on $0.6 \mathrm{U} / \mathrm{ml}$ of proteinase $\mathrm{k}$ (Promega Corporation, Madison, WI) for 30 minutes at room temperature, blocking nonspecific binding with $10 \%$ new-calf serum blocking reagent (Sigma-Aldrich, St. Louis, MO). The primary antibody in $0.1 \%$ bovine serum albumin (SigmaAldrich) and $0.4 \%$ Triton-X (Sigma-Aldrich) was applied overnight at $4^{\circ} \mathrm{C}$. After secondary $\mathrm{Ab}$ (Vector Laboratories) incubation and quenching, slides were incubated with Vectastain $A B C$ (Vector Laboratories) as per manufacturer's instructions with color development using NovaRED (Vector Laboratories). Slides were counterstained with Harris hematoxylin (Bio Optica, Milano, Italia). Negative control specimens were stained with a rabbit isotypic IgG primary Ab (Vector Laboratories). Stained slides were examined using a Zeiss Axioskop (Carl Zeiss, Milano, Italy) with either a $10 / 0.25$ NA or $40 / 0.6$ NA dry objective. Photomicrographs were acquired with an Axiocam-IcC3 color camera and Axiovision-4.8.2 software (Carl
Zeiss). Quantification of HOXB7 positive stained area was performed using Image J software (http://rsb.info.nih.gov/ij/).

\section{Isolation and Ex Vivo Expansion of MSC}

BM specimen harvests were performed from posterior iliac crest following standard procedure. All MSC samples were isolated, amplified, and cumulative population doubling (CPD) was calculated as previously reported $[25,26]$. RNA extraction, immunophenotypical analyses, and differentiations were performed after four passages (P4), as below described.

\section{Fluorescence-Activated Cell Sorting Analyses}

Immunophenotype of ex vivo expanded MSC was performed as reported [27]. The following panel of monoclonal antibodies was introduced, CD14-APC, CD73-PE, CD105-PE (all from BD Pharmingen, San Diego, CA), CD90-APC (eBioscience, San Diego, CA), FGFR1-APC (R\&D Systems, Minneapolis, MN), and analyzed with FACS-ARIA (Becton Dickinson, Franklin Lakes, NJ). Collected data were elaborated by fluorescence-activated cell sorting (FACS) Diva software (Becton Dickinson).

\section{Human MicroRNA Expression Detection}

RNAs from MSC samples were hybridized on Agilent Human miRNA microarray (\#G4470B, Agilent Technologies, Palo Alto, CA). This microarray consists of 60-mer DNA probes synthesized in situ and contains 15,000 features which represent 723 human microRNAs, sourced from the Sanger miRBASE public database (Release 10.1). One-color miRNA expression was performed according to the manufacturer's procedure. Briefly, total RNA fraction is obtained from samples using the Trizol Reagent (Invitrogen, Carlsbad, MN). RNA quality is assessed using the Agilent 2100 Bioanalyzer (Agilent Technologies). Low quality RNAs (RNA integrity number below 7) were excluded from microarray analyses. RNA labeling and hybridization were performed in accordance to manufacturer's indications as detailed in [28]. Agilent scanner and the Feature Extraction 10.5 software (Agilent Technologies) were used to obtain the microarray raw-data.

\section{Microarray Data Analysis}

Microarray results were analyzed using the GeneSpring GX 11 software (Agilent Technologies). Data transformation was applied to set all the negative raw values at 1.0, followed by quantile normalization. A filter on low gene expression was used to keep only the probes expressed in at least one sample (flagged as Marginal or Present). Then, samples were grouped in accordance to their differentiation status and compared. Differentially expressed genes were selected as having a 1.5-fold expression difference between the groups of interest and a statistically significant $p$-value $(<.05)$ by unpaired $t$ test.

\section{Quantitative Real-Time RT-PCR}

Total RNA from MSC cultures was harvested by Trizol (Invitrogen). cDNAs of total RNA fraction were generated with random hexamers using RevertAid First Strand cDNA Synthesis Kit (Fermentas, Thermo Fisher Scientific, Waltham, MA), following the manufacturer's recommendations. RNA and cDNA were quantified with Beckman Coulter spectrophotometer. Ten nanograms of small RNA input was reverse transcribed using RevertAid First Strand cDNA Synthesis Kit (Fermentas, Thermo Fisher Scientific) together with specific primers (Ambion, Van Alley Way, CA). 
PCR reactions to quantify microRNA expression were prepared using TaqMan MicroRNA Assays (Ambion) with TaqMan Universal Master Mix II, no UNG (Applied Biosystems, Thermo Fisher Scientific). RNU24 was used for normalization. PCR reactions to quantify mRNA expression were prepared using fast SYBR green master mix (Applied Biosystems, Thermo Fisher Scientific). $\beta$-Actin was used for normalization. Quantitative PCR was performed on Step One Real-Time PCR System (Applied Biosystems, Thermo Fisher Scientific). Primers are listed in Supporting Information Table S1. All measurements were performed in triplicate and analysis was made by Step One software using $\Delta \mathrm{Ct}$ method [29].

\section{miRNA Target Site Prediction}

A search for predicted target miRNAs was performed using the database TargetScan (http://www.targetscan.org/), PicTar (http://pictar.mdc-berlin.de/), and DIANA microT v3.0 (http:// diana.cslab.ece.ntua.gr/microT/).

\section{Dual Luciferase Reporter Gene Construct}

A 469-bp fragment of the HOXB7 $3^{\prime}$ UTR containing the predicted binding site for has-miR-196a was amplified from human total RNA using primers with a short extension containing cleavage sites for Sacl ( $5^{\prime}$ end) and Xbal ( $3^{\prime}$ end): HOXB7-3'UTR forward ( $5^{\prime}$ tatcgagct/cgAGAAGCCCAGCTCTGGGAA C-3') and HOXB7-3'UTR reverse ( $5^{\prime}$-tatct/ctagagCCAGGCGCTTGGGGAACAC- $\left.3^{\prime}\right)$. Amplicons were cleaved with Sacl and $\mathrm{Xbal}$ and cloned in between the Sacl and Xbal cleavage sites of the pmiRGLO vector (Promega, Madison, WI) downstream of the Firefly luciferase reporter gene. The delete form of HOXB7-3'UTR, $\triangle H O X B 7-3^{\prime} U T R$, lacking miR-196a complementary sequence (TTCAACACTGAAAAAATACTACCTA) has been created by SOE-PCR technique [30], using additional primers ( $\Delta$ forward: 5'-AAATTTTGGTTTTAGGAA AGTCTGTCAGGTTTGGTTTTTTTTG-3'; $\Delta$ reverse $5^{\prime}$-CAGACTTT CCTAAAACC AAAAT TTCTCСTTTCTCССТCC-3').

\section{Luciferase Assay}

Primary MSC were cotransfected by Nucleofector $2 b$ device (Lonza, Basel, Switzerland) with $50 \mathrm{nM}$ pre-miR-196a or miR control (Ambion, Van Alley Way, CA) and $100 \mathrm{ng}$ of pmiRGLO vector, pmiRGLO-HOXB7-3'UTR, or the delete form using Amaxa Human MSC Nucleofector Kit (Lonza) as per manufacturer's instructions. Firefly and Renilla luciferase were measured after 28 hours in cell lysates using a Dual-Luciferase Reporter Assay System (Promega) on a Victor Light plate reader (PerkinElmer, Waltham, MA). Renilla luciferase activity was used for normalization and as an internal control for transfection efficiency.

\section{Viral Infection with MigRI-HOXB7 Vector}

HOXB7 coding sequence (NM_004502.3) was amplified from human total RNA using primers with a short extension containing cleavage sites for BgllI ( $5^{\prime}$ end) and EcoRI ( $3^{\prime}$ end): HOXB7 forward (5'-TATCAGATCTAAATCATCCGGCCAAATTATGAG$\left.3^{\prime}\right)$ and HOXB7 reverse (5'-TATCGAATTCTGCCCTTTCTCCATCCCTC $\left.A C-3^{\prime}\right)$. Amplicons were cleaved with BgIII and EcoRI and cloned between the Bglll and EcoRI cleavage sites of the MigR1 (MSCV-GFP) vector. The resulting vector was defined as MIGR1-HOXB7, whereas the empty MIGR1-GFP vector was used as control. Retrovirus production was performed by the FLYRD18 packaging cell lines and three different BM-MSC samples were then transduced by virus-containing media from either FLYRD18-HOXB7 or FLYRD18-GFP (both $\sim 1 \times 10^{5}$ transducing units $/ \mathrm{ml}$ ), as described [31]. The obtained MSC lines were defined as MSC1-HOXB7, MSC1-GFP, MSC2-HOXB7, MSC2-GFP, MSC3HOXB7, and MSC3-GFP, respectively. MSC cell lines were analyzed by Western blot to check HOXB7 overexpression. Protein extracts were prepared with direct lysis in Laemmli sample buffer (125 mM TRIS HCl pH 6.8, 20\% glycerol, 4\% SDS, Bromophenol $0.04 \%$ Blue). Protein from each extract was fractionated in a SDSpolyacrylamide gel (Bio-Rad, Hercules, CA) and then transferred to nitrocellulose. HOXB7 detection was performed by a rabbit anti-HOXB7 polyclonal as primary antibody (Invitrogen) and goat anti-rabbit IRDye800CW (LI-COR, Lincoln, NE) as secondary antibody. Normalization of protein loading was performed with antiGAPDH antibody (Cell Signaling Technology, Danvers, MA). The signal was captured by Odyssey infrared imaging system (LI-COR).

\section{Clonogenic Assay}

Triplicate samples of MSC were seeded at 100 cells per square centimeter in culture media as described [26]. On day 10 , cells were fixed with cooled absolute methanol for 2 minutes and stained for 5 minutes with $1 \%$ crystal violet aqueous solution (Sigma-Aldrich). Colonies with more than 50 cells were then scored and cloning efficiency $(E)$ was calculated as $E \%=(n$ clones/cell seeded $) \times 100$.

\section{Beta-gal Staining}

Expression of $\mathrm{pH}$-dependent senescence associated $\beta$ galactosidase (SA- $\beta$-gal) activity was analyzed in the gene modified samples of MSC using the SA- $\beta$-gal staining kit (Cell Signaling Technology). Positive cells were counted considering seven fields for each sample.

\section{Karyotypic Evaluation}

Cytogenetic analyses were performed at passage 13 or higher as described [32].

\section{Differentiation Assay}

Three lineages differentiation potential of MSC was assessed as reported [25]. In particular for osteogenic induction, MSC were seeded in conditioned medium with Dulbecco's modified Eagle's medium (Euroclone, Milano, Italy) containing 10\% Fetal Bovine Serum (FBS) (Hyclone, Logan, UT), 1\% P/S $\left(10^{4} \mathrm{UI} / \mathrm{ml}\right.$ and $10 \mathrm{mg} / \mathrm{ml})$, and glutamine $(2 \mathrm{mM})$ supplemented with dexamethasone (10 nM), L-ascorbic acid-2-phosphate (0.1 mM), beta-glycerol phosphate $(2 \mathrm{mM}$ ) (all from SigmaAldrich), and bone morphogenic protein (BMP)-2 (100 ng/ $\mathrm{ml}$; Tebu-Bio, Magenta, Italy) for 2 weeks. Osteogenic differentiation was assessed by von Kossa staining on cultured cells that were fixed on ice cold methanol for 2 minutes, rinsed in distilled water, and incubated with $1 \%$ silver nitrate for 30 minutes under a UV lamp. Stained samples were then washed and visualized by $\times 10$ magnification using an inverted microscope (Zeiss). The percentage of Von Kossa stained dark area was calculated by Image J software (http://rsb.info.nih.gov/ij/) [26].

\section{ELISA}

Basic fibroblast growth factor (bFGF) levels on gene modified MSC were measured by Quantikine Human bFGF kit (R\&D Systems) according to manufacturer instructions. 
A
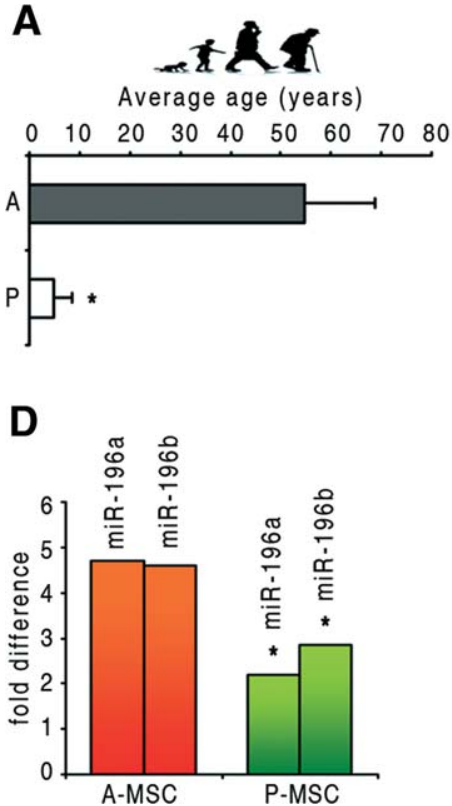

B

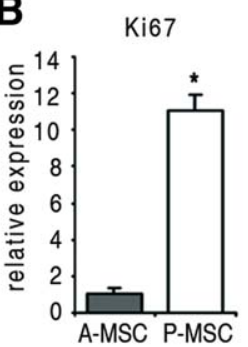

E

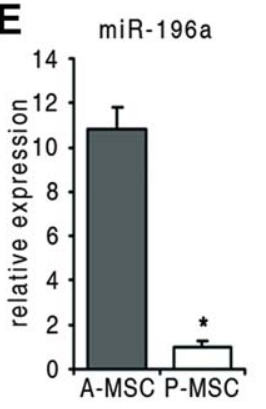

C

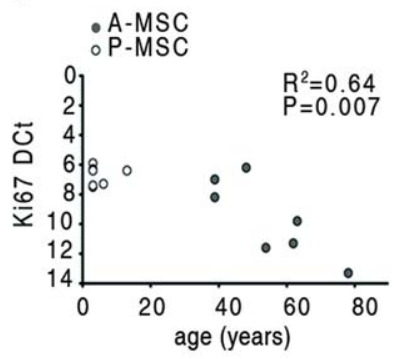

$\mathbf{F}$

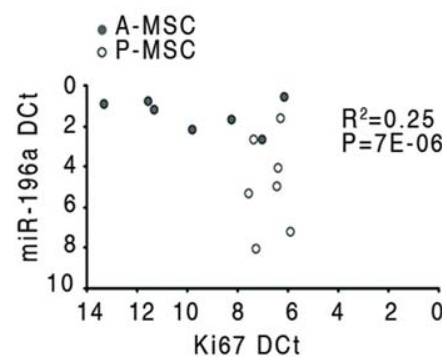

Figure 1. Ki-67 expression is age repressed in human MSC and negatively correlates with miR-196 levels. (A): Average age of adult (A) and pediatric $(\mathrm{P})$ groups; ${ }^{*}, p=1.1 \times 10^{-6}$. (B): Ki67 mRNA expression in MSC from adult (A-MSC) and pediatric (P-MSC) groups; *, $p=.01$. (C): Correlation analysis between age and Ki67 DCt; lower values of DCT correspond to higher Ki67 expression. (D): Relative miR-196a and miR-196b levels in A-MSC $(n=4)$ and P-MSC $(n=3)$ identified by microarray analyses; *, $p=.04$. (E): Validation of miR196a expression performed by quantitative RT-PCR in A-MSC $(n=7)$ and P-MSC $(n=7) ; *, p=.003$. (F): Correlation analysis between miR-196a levels and Ki67 mRNA expression in A-MSC and P-MSC. All $p$ values by unpaired two-tailed Student's $t$ test. Error bars, SEM. Abbreviations: A-MSC, adult mesenchymal stromal/stem cells; P-MSC, pediatric mesenchymal stromal/stem cells.

\section{FGFR Inhibition}

MSC were seeded at 7,000 cells per square centimeter and, after 24 hours, PD166285 (Calbiochem, Merck Chemicals International, Darmstadt, Germany) in DMSO was added to culture media at final concentration of $55 \mathrm{nM}$. PD166285 was added again at 48 and 72 hours. DMSO only was used as control. After 96 hours from seeding, cells were harvested for molecular studies.

\section{Statistical Analyses}

Data are presented as mean \pm SEM. Comparisons and correlations were made by two-tailed $t$ test and Pearson's, respectively, by Microsoft Excel 2010 with values considered statistically significant at $p<.05$.

\section{RESULTS}

\section{Pediatric and Adult MSC Significantly Differ for Ki-67 Expression in an Age-Dependent Manner}

Adult group (A) consisted of two females and five males ranging from 39 to 78 years-old, while pediatric group (P) considered subjects ranging from 3 to 13 years-old and was composed by four females and three males. There was a significant difference in the average age between adults $(54.7 \pm 14.1)$ and pediatric donors (4.8 \pm 3.7$)$ (Fig. 1A). Having defined the age as a highly discriminating parameter, we began to assess the proliferative potential of A-MSC and P-MSC using Ki67, a well established index of cell proliferation capacity [33]. Ki67 expression is significantly higher in pediatric samples (Fig. 1B) showing an inverse correlation with donor age (Fig. 1C). These findings confirm at the molecular level the greater proliferative potential of MSC from pediatric donors [5, 7-10].

\section{Pediatric MSC Reveal a Distinct miRNA Profile}

In order to understand whether the observed different proliferative potential of P-MSC was linked to distinct miRNA expression, the groups were further compared considering platform of 723 miRNA. From these analyses, we were able to identify only seven differentially expressed miRNA between the two groups: miR-99a, miR-100, miR-196a, miR-196b, miR-337-5p, miR-376b, miR-431, and miR-543 ( $p<.04$; data not shown). Among those, both miR-196a and miR-196b resulted less expressed in P-MSC (Fig. 1D). While having overlapping sequences except for one nucleotide, isoforms $a$ and $b$ of miR-196 derive from three different chromosome loci (chromosomes 17 and 12 for isoform a, chromosome 7 for isoform b) and share predicted targets [34]. In addition, miR-196a seems to impact on differentiation and proliferation of human adipose-derived MSC [35], therefore we focused on the a isoform only. To validate microarray findings, quantitative real-time RT-PCR (qRT-PCR) revealed a consistently lower miR-196a expression in P-MSC (Fig. 1E) whose level was inversely correlated with Ki67 (Fig. 1F), indicating a link between the proliferative attitude of P-MSC and the identified miRNA.

\section{HOXB7 Expression Is Regulated by miR-196a in MSC}

In silico analysis revealed several HOX genes as predicted miR-196a targets (not shown). During prenatal development HOX regulate neuro-ectodermal and mesodermal progenitors proliferation and fate $[36,37]$; therefore, we hypothesized a role of this family in the observed proliferative events of postnatal mesenchymal progenitors in relationship with miR-196a. Among HOX targets we focused on HOXB7, since its 
A

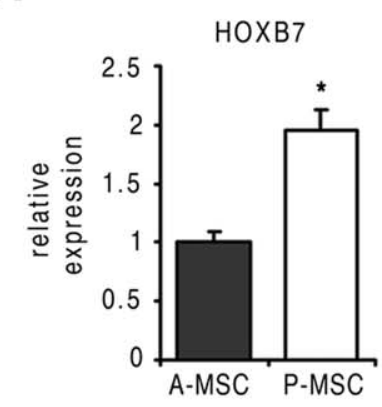

B

- A-MSC

- P-MSC

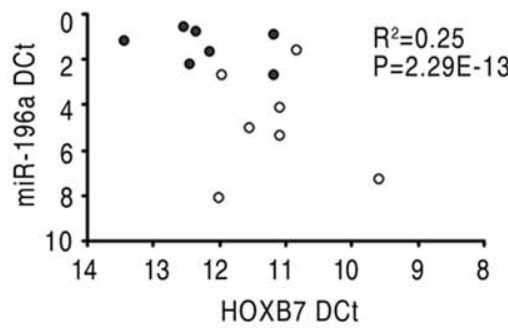

C

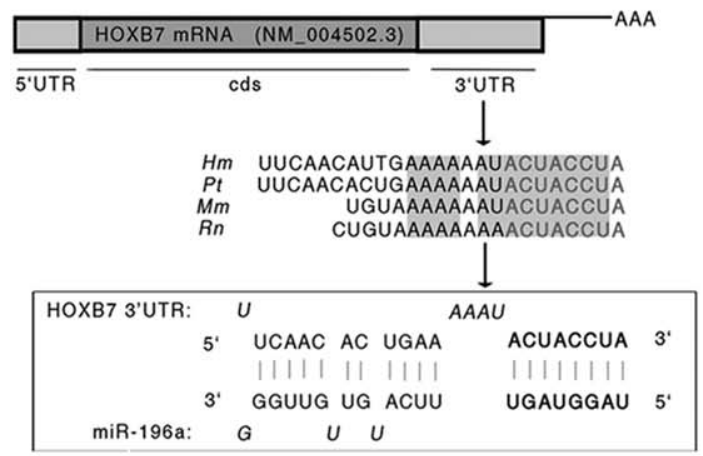

D

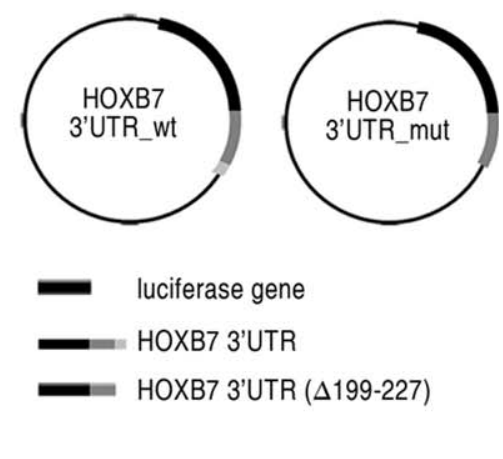

E

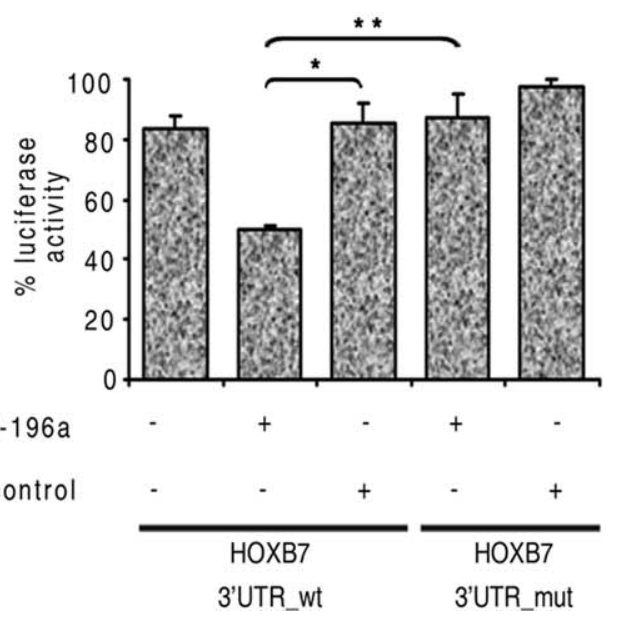

Figure 2. HOXB7 is a direct target of miR-196a in MSC. (A): HOXB7 mRNA expression in A-MSC $(n=7)$ and P-MSC $(n=7) ; *, p=.04$. (B): Correlation analysis between miR-196a and HOXB7 mRNA levels in A-MSC and P-MSC. (C): Diagram illustrating HOXB7 $3^{\prime} U T R$. In gray the conserved sequence among species. In the box the predicted consequential pairing of $3^{\prime} U T R$ target region (top) and miR-196a (bottom). Seed region in bold. (D): Schematic representation of luciferase reporters with the wild-type HOXB7 $3^{\prime} U T R$ (HOXB7_3'UTR_wt) or the mutated HOXB7 $3^{\prime}$ UTR lacking of the whole $(\Delta 199-227)$ miR-196a complementary sequence (HOXB7_3'UTR_mut). (E): The effect of pre-miR-196a and pre-miR negative control (miR control) on luciferase activity in MSC transfected with the HOXB7_3'UTR_wt reporter (lanes 1-3) or the HOXB7 3'UTR mut reporter (lanes 4 and 5). ${ }^{*}, p=.0007$ and ${ }^{* *}, p=.001$. Experiments were performed in triplicate. All $p$ values by unpaired two-tailed Student's $t$ test. Error bars, SEM. Abbreviations: A-MSC, adult mesenchymal stromal/stem cells; Hm, Homo sapiens; Mm, Mus musculus; P-MSC, pediatric mesenchymal stromal/stem cells; Pt, pan troglodytes; Rn, Rattus norvegicus.

expression has been previously related with cell aging and senescence $[14,16]$. Comparing groups, we observed a significantly higher expression of HOXB7 in P-MSC (Fig. 2A) and an inverse correlation between miR-196a and HOXB7 levels (Fig. $2 \mathrm{~B})$, indicating a link between HOXB7 and donor age and suggesting an interplay between the HOXB7 and miR-196a.

The relationship between miR-196a and HOXB7 in MSC was then explored by miRNA mimic and inhibitor assays as shown in Supporting Information Figure S1. While miR-196 mimic determined the reduction of HOXB7 level (Supporting Information Fig. S1A), conversely miRNA inhibition resulted in its upregulation (Supporting Information Fig. S1B).

To ultimately verify whether HOXB7 is a target of miR$196 a$ in our system, direct targeting of the $3^{\prime}$ UTR region of the HOXB7 gene was then challenged by a dual luciferase reporter assay. HOXB7 has 8-nt seed match site for miR-196a 
A

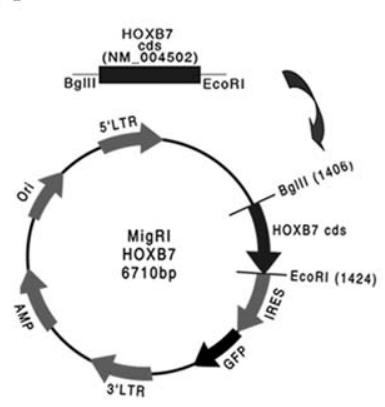

B

D
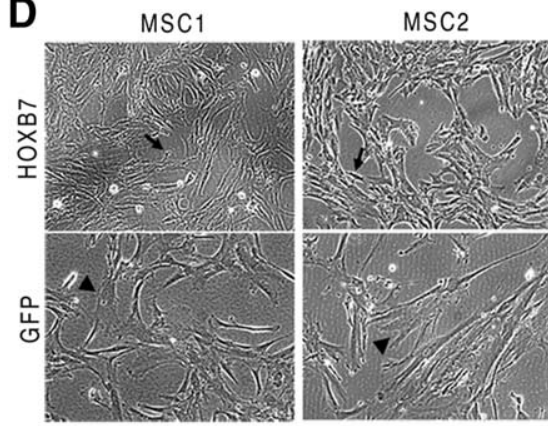

F

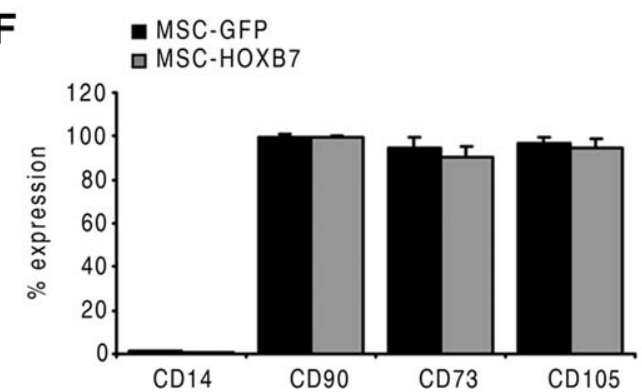

C

E MSC-GFP
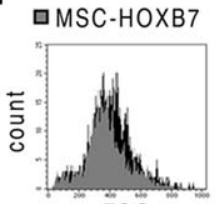

FSC
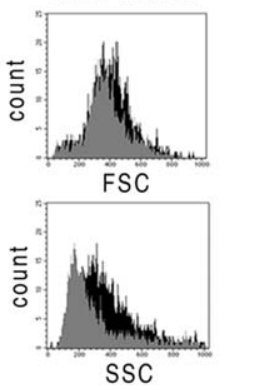
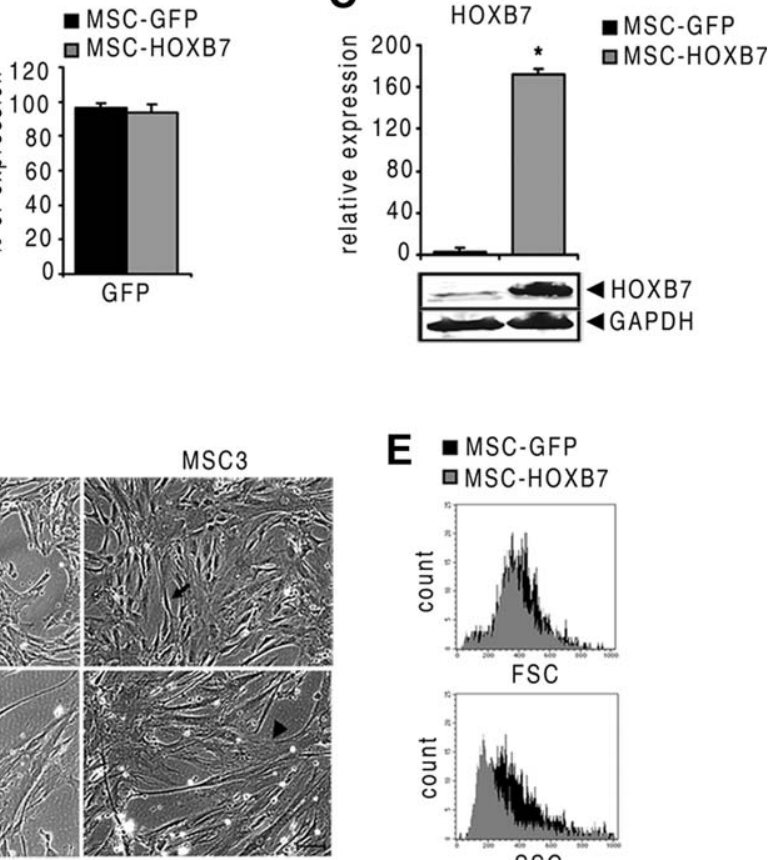

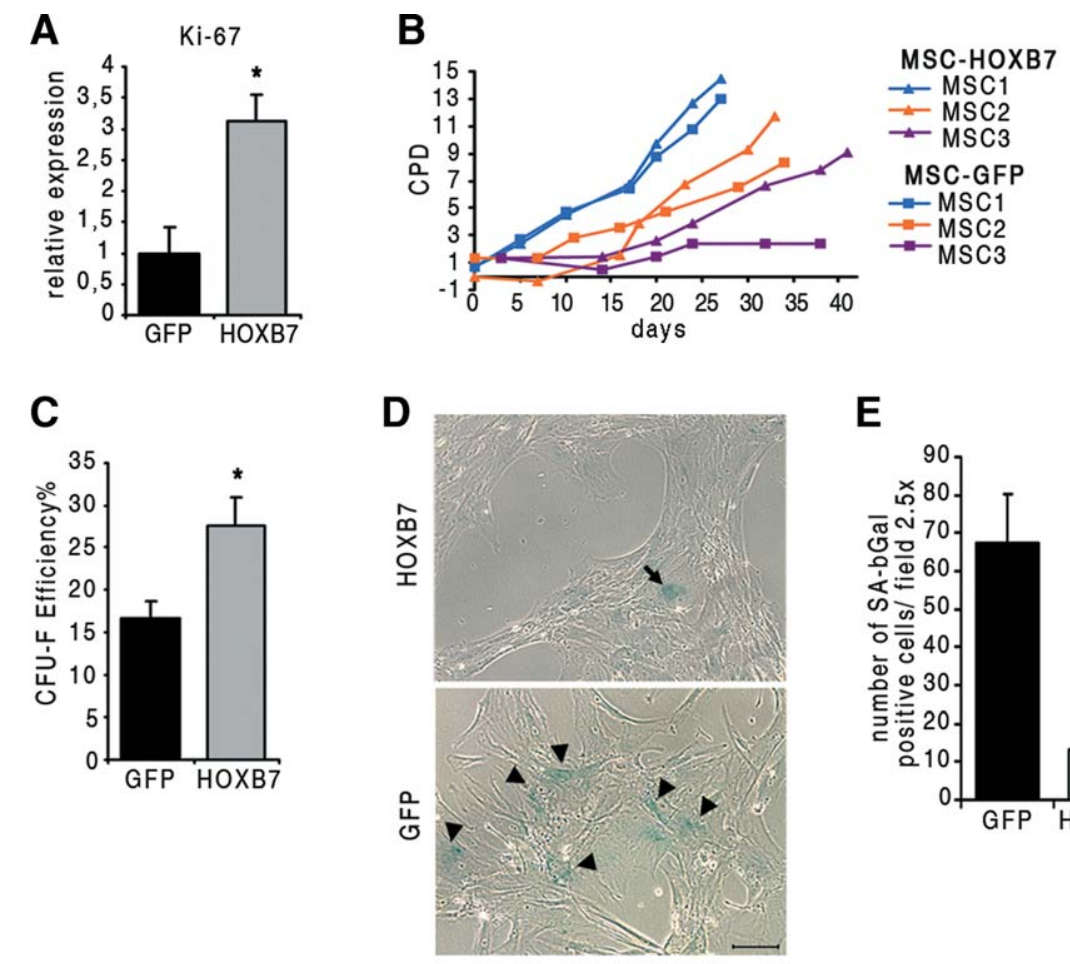

E
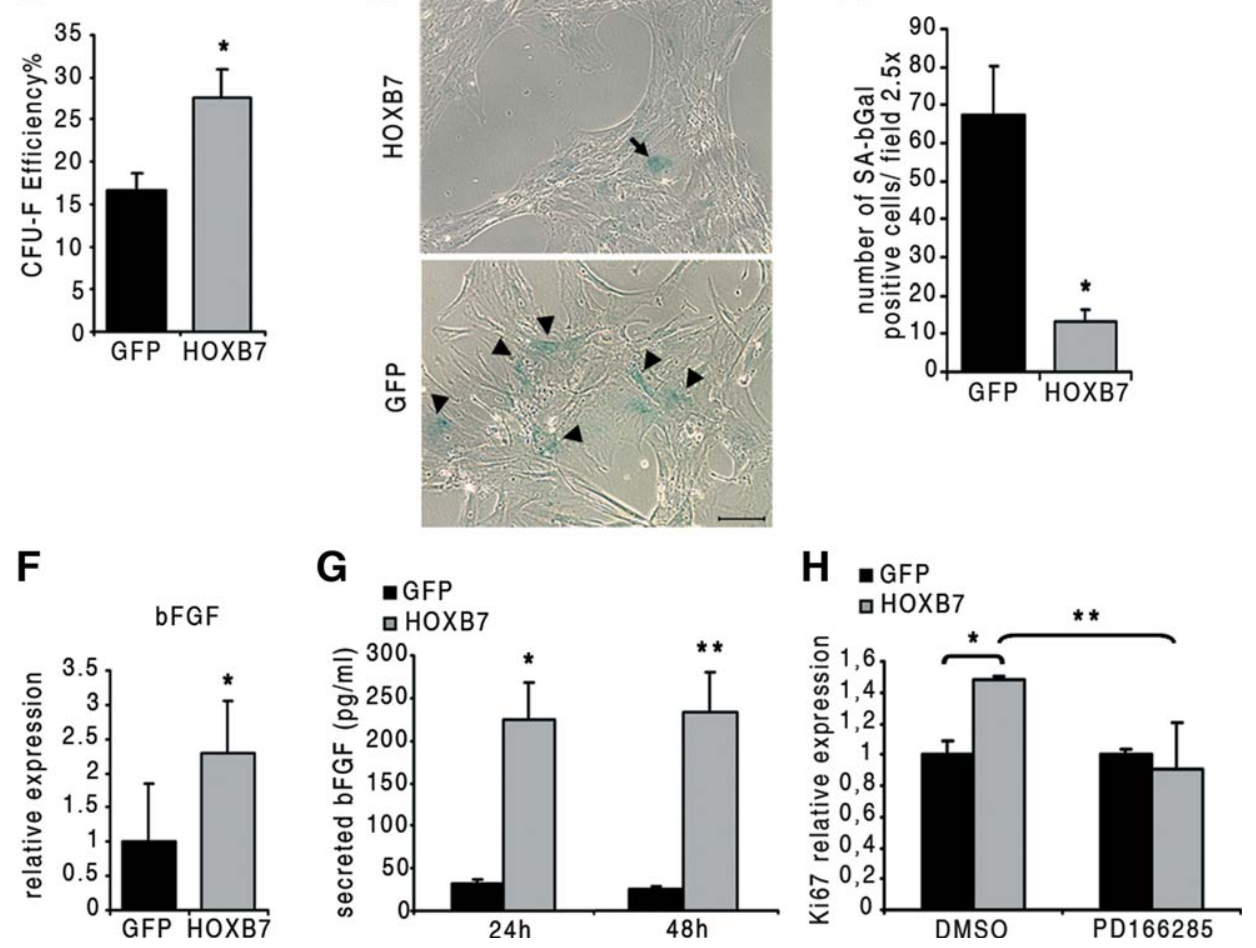

Figure 4. MSC-HOXB7 retain higher in vitro performance combined with reduced senescence linked with increased levels of secreted bFGF. (A): Ki67 mRNA fold increase in MSC-HOXB7 compared to MSC-GFP at passage 10, $*, p=.039$. (B): Cumulative population doublings of MSC-HOXB7 and MSC-GFP calculated from passage 3 to passage 9. (C): Colony assay at passage 14 on a representative sample (MSC1) to evaluate clonogenic potential of MSC-GFP and MSC-HOXB7. E\% $=(n$ clones/cell seeded $) \times 100, *, p=.009$. (D): Representative SA- $\beta$-galactosidase staining of MSC-HOXB7 (upper panel) and MSC-GFP (lower panel) performed at passage 13 . Scale bar $=100 \mu \mathrm{m}$. (E): Quantification of SA-b-gal positive cells $/ \times 2.5$ high power field $(n=7)$. ${ }^{*}, p=.0004$. (F): bFGF mRNA fold increase in MSC-HOXB7 compared to MSC-GFP at passage 12. Data are the mean of three biological samples $*, p=.00002$. (G): Secreted bFGF in supernatant of MSC-GFP and MSC-HOXB7 after 24 and 48 hours of culture. Data are the mean of three biological samples $*, p=.002$ HOXB7 versus GFP at 24 hours and **, $p=.009$ versus GFP at 48 hours. (H): Ki67 expression after in vitro treatment (96 hours) with FGFR-1 inhibitor (PD166285) in both MSC-GFP (P18) and MSC-HOXB7 (P16). ${ }^{*}, p=.0006$ and ${ }^{* *}, p=.02$. All $p$ values by paired two-tailed Student's $t$ test. Error bars, SEM. Abbreviations: bFGF, basic fibroblast growth factor; GFP, green fluorescent protein; MSC, mesenchymal stromal/ stem cells.

reported criteria to assess MSC senescence [9]. MSC-HOXB7 reduced their size (FSC) with a lower internal complexity (SSC), suggesting a diminished in vitro aging versus the MSC-GFP (Fig. $3 \mathrm{E})$. Regarding surface markers, MSC immunophenotype was not influenced by HOXB7 (Fig. 3F). Collectively, these data indicate that extensively amplified MSC overexpressing HOXB7 reveled distinct morphological changes in vitro associated with a persistence of the typical surface fingerprints of MSC.

\section{HOXB7 Overexpression Is Associated with Higher Proliferative Potential and Reduced Senescence}

In the attempt to verify the hypothesis of an improved cell performance due to forced expression of HOXB7, in vitro expansion was monitored by Ki67 analysis. In MSC-HOXB7, we observed a higher expression of the proliferation marker compared to MSC-GFP (Fig. 4A), suggesting an impact of this HOX in regulating adult MSC proliferation. Interestingly, HOXB7 overexpression was associated with downregulation of miR196a levels as shown in Supporting Information Figure S2. Long-term in vitro propagation studies were then introduced to assess the CPD of transduced cells. Once more, MSCHOXB7 samples were associated with a better proliferative performance compared to the relative green fluorescent protein (GFP) control (Fig. 4B) without cytogenetic alterations (not shown). Additionally, the effect of HOXB7 on MSC clonogenic activity was assessed by CFU-F assay, showing that MSC-HOXB7 were generating a higher number of colonies (Fig. 4C). The greater cloning efficiency and proliferative 
A

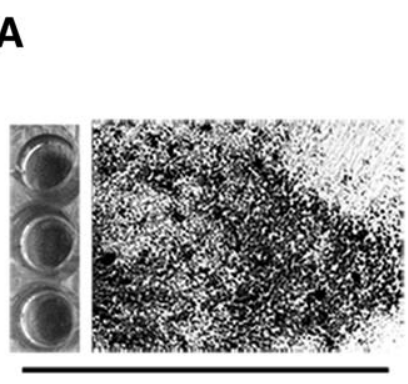

HOXB7 induced

B

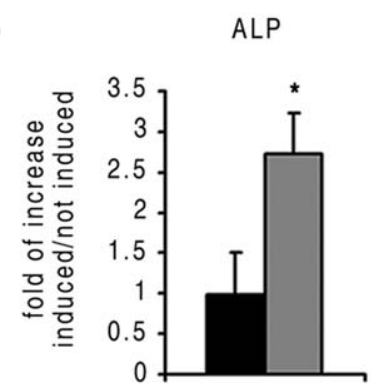

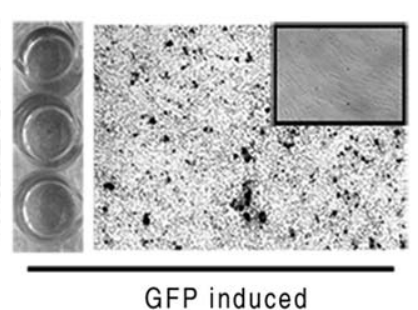

GFP induced
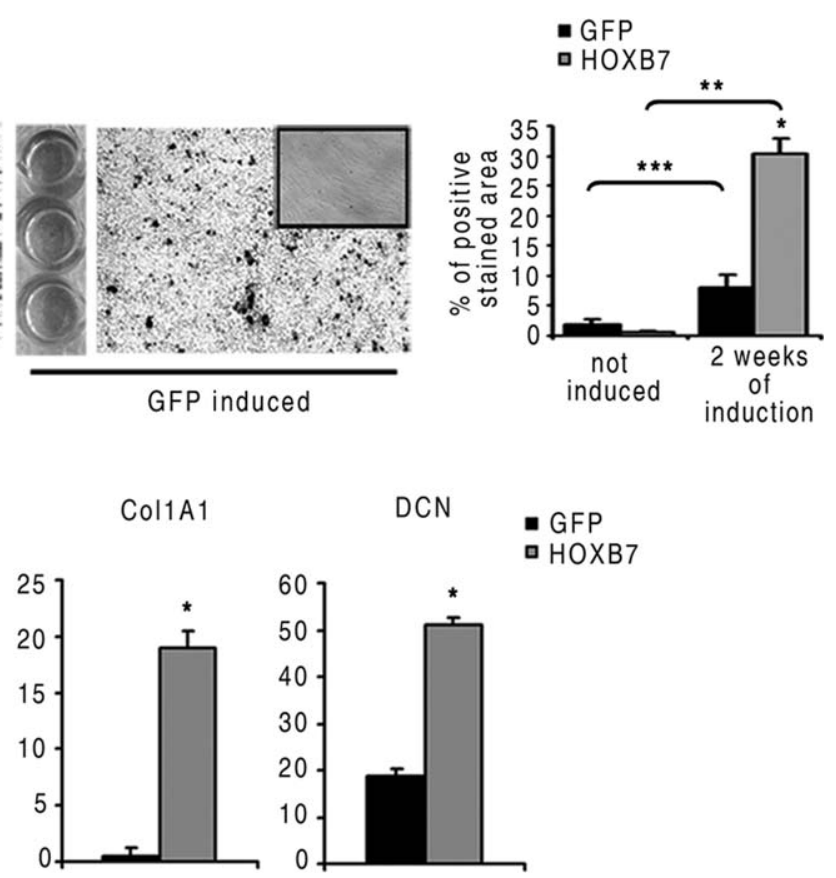

- GFP

GOXB7

Figure 5. Mesenchymal stromal/stem cells (MSC)-HOXB7 display a higher osteogenic potential compared to MSC-GFP. (A): Von Kossa staining after osteogenic induction of MSC-GFP and MSC-HOXB7 at passage 12 in multiwell plates. Not induced control in the inset panel. Quantification by Image J of positive stained area on the right ${ }^{*}, p=3.9 \times 10^{-51}$ HOXB7 versus GFP at 2 weeks of induction, ${ }^{* *}, p=1.6 \times 10^{-23}$ and $* * *, p=6.5 \times 10^{-10}$. (B): mRNA expression of osteogenic markers ALP, Col1A1, and DCN. Histograms show the fold of increase after induction in MSC-GFP and MSC-HOXB7. ${ }^{*}, p=.03 ;{ }^{*}, p=.02 ;{ }^{*}, p=.01$, respectively. Data are the mean of three biological samples. All $p$ values by paired two-tailed Student's $t$ test. Error bars, SEM. Abbreviations: ALP, alkaline phosphatase; Col1A1, collagen type1 alpha1; DCN, decorin; GFP, green fluorescent protein.

potential of MSC-HOXB7 were then also considered in terms of senescence, measuring cellular aging in vitro using a $\beta$ galactosidase ( $\beta$-Gal) staining [9]. Confirming morphological changes and growth curves of MSC-GFP, the staining outlined a significantly higher quote of $\beta$-gal positive cells in these latter samples (Fig. 4D, 4E, arrow heads), indicating that HOXB7 improves MSC performance increasing proliferation and reducing senescence.

\section{HOXB7 Generates a bFGF-Mediated Autocrine Loop in MSC}

In order to provide an understating of the proliferative attitude of MSC-HOXB7, we focused on bFGF as a pivotal MSC mitogen and known transcriptional target of HOXB7 in cancer [38]. Measuring bFGF levels in modified MSC, we observed that bFGF mRNA was highly expressed in MSC-HOXB7 (Fig. $4 \mathrm{~F})$. These data were then confirmed measuring secreted bFGF that was significantly increased in MSC-HOXB7 supernatants after both 24 hours and 48 hours of culture (Fig. 4G). To further explore whether FGF signaling was effectively responsible of HOXB7 action on proliferation, we assessed FGFR1 expression on modified MSC, observing comparable levels between MSC-GFP and MSC-HOXB7 (Supporting Information Fig. S3). Finally, using PD166285, a known inhibitor of FGFR1 tyrosine kinase [39], we wanted to verify if FGF signaling blockage could abrogate the MSC-HOXB7 proliferative advantage. After 4 days of treatment with PD166285, the expression of Ki67 became comparable to the control MSC-GFP, indicating that a specific inhibition of FGF signaling is able to revert the increased proliferative potential due to HOXB7 overexpression (Fig. 4H), further outlining HOXB7 as influential player on in vitro behavior of human primary stem cells by
bFGF autocrine loop. To further explore the impact of HOXB7 on MSC, a loss of function experiment was introduced. The reduction of HOXB7 expression was associated with decreased bFGF and Ki67 levels as shown in Supporting Information Figure S4, confirming the relationship among these players and the role of HOXB7 on MSC proliferation.

\section{HOXB7 Specifically Enhance MSC Osteogenic Potential}

Since bFGF levels are associated with an increase MSC proliferation coupled with osteogenesis in vivo $[24,40]$ and $\mathrm{HOX}$ are linked with prenatal skeletal development [34, 37, 41], we evaluated whether HOXB7 overexpression may be associated by an increased mineralizing potential in extensively amplified MSC. Thus, a BMP-2 in vitro assay was introduced and, after 2 weeks of induction, MSC-HOXB7 showed an impressive von Kossa staining versus the induced MSC-GFP with more of threefold increase in osteogenic commitment (Fig. 5A), while no apparent differences could be detected in inducing MSCHOXB7 toward chondrogenic and adipogenic lineages (Supporting Information Fig. S5). A clustered gene expression analysis outlined an increase of pivotal osteogenic-related biomarkers in MSC-HOXB7 versus MSC-GFP (Fig. 5B), supporting the role of HOXB7 in bone formation, further suggesting that the observed HOXB7 levels in osteoprogenitors from pediatric population may be linked to higher osteogenesis in the early phase of postnatal life.

\section{HOXB7 Expression in Skeletal Tissues Is Age Related in Mice}

To explore this latter hypothesis, we considered HOXB7 levels into skeletal tissue of mice aged 11,90 , and 180 days. At the 
A

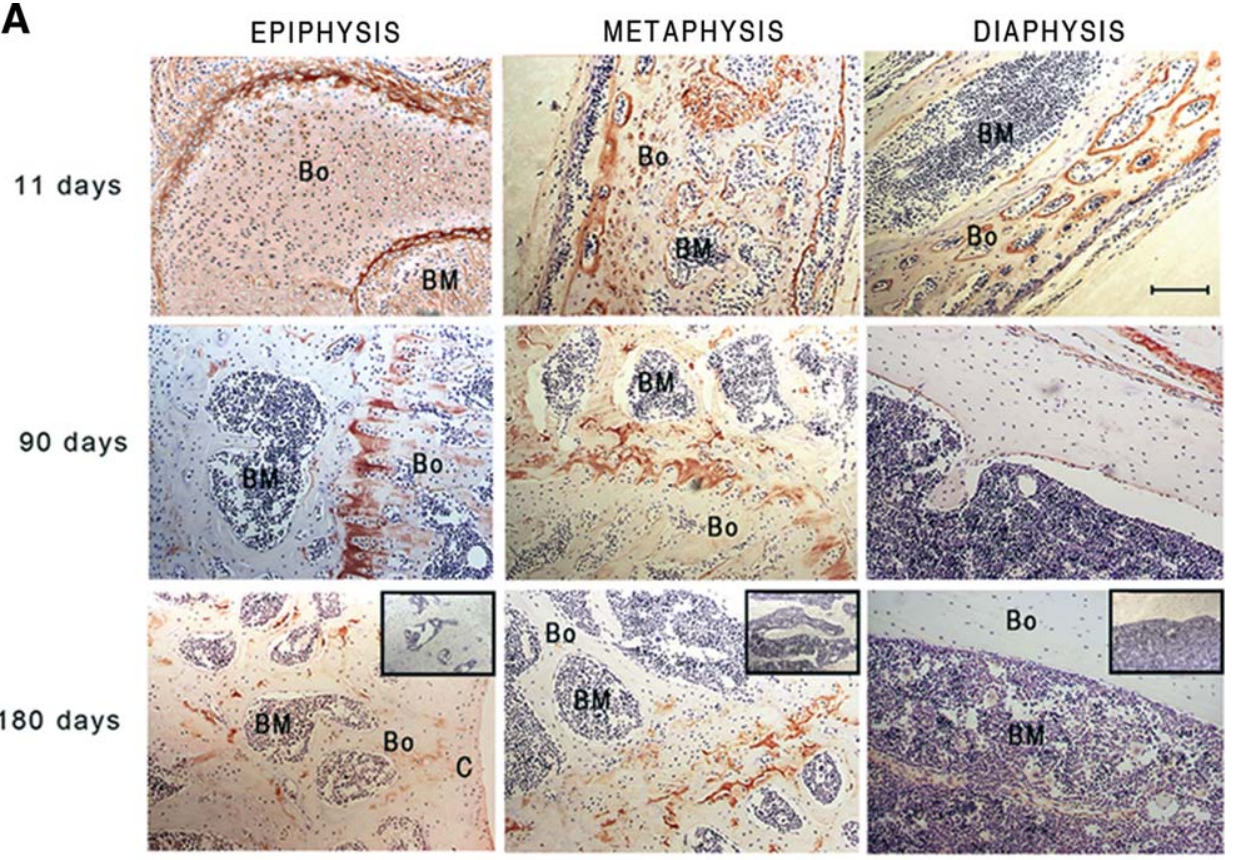

B

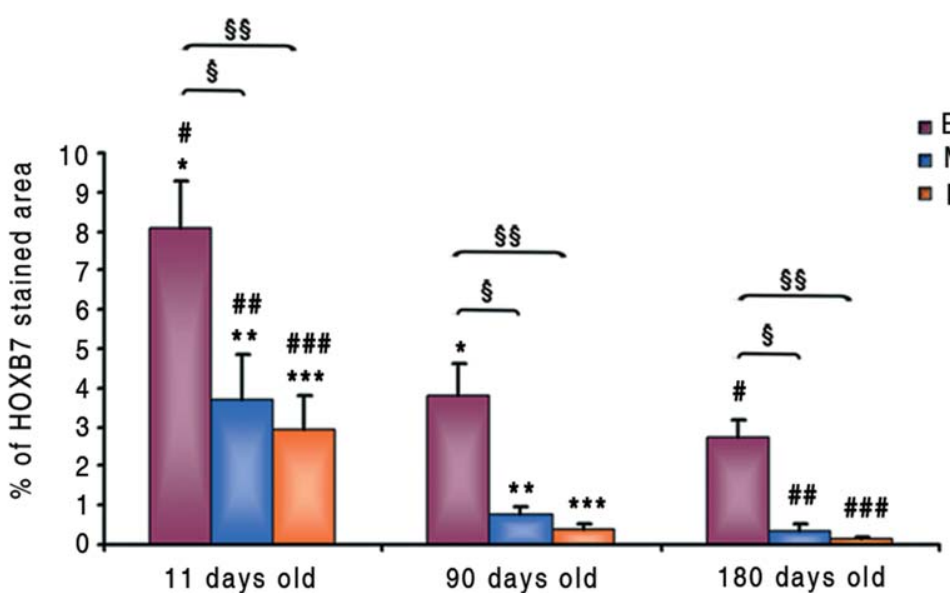

Figure 6. HOXB7 levels decreases in vivo during aging. (A): Anti-HOXB7 antibody IHC staining of murine bone from 11, 90 , and 180 days old mice. Staining with isotypic IgG antibody (negative control) in inset panels. Representative images are shown. Scale bar $=100$ $\mu \mathrm{m}$. (B): Quantification (by ImageJ) of HOXB7 positive stained area at epiphyseal, metaphyseal, and diaphyseal levels of murine bone. Three mice were analyzed, $n=3$ ( $n$ of fields $=3$ for each animal). All $p$ values indicated by symbols $*, * *, * * *, \#, \# \#, \# \#, \S, \S \S$ are $<.05$ by unpaired two-tailed Student's $t$ test. Error bars, SEM. Abbreviations: BM, bone marrow; Bo, bone; C, cartilage.

early time point, the immunohistochemical studies revealed a relevant expression of HOXB7 into the different bone regions, having the epiphyseal areas the highest expression (Fig. 6A, upper row). At 90 days, while stained cells could be still detected at the growth plate as well as in the endosteal and periosteal areas, a progressive reduction of HOXB7 staining was evident (Fig. 6A, middle row). This pattern further changed at 180 days with a dramatic decline of HOXB7 expression that became undetectable at cellular level while remaining into the matrix (Fig. 6A, lower row). To quantify the histological staining, we measured the percentage of HOXB7 positive stained tissue, demonstrating a progressive and significant reduction of HOXB7 during aging and confirming a preferential expression at epiphyseal level for all time points (Fig. 6B). To verify the specificity of HOXB7 staining additionally addressing the expression of another HOX factor
[42], HOXB9 expression was assessed. Murine bone did not express HOXB9 which was instead limited to articular cartilage in newborn mice and was almost undetectable in 90 and 180 days old mice (Supporting Information Fig. S6) indicating that besides the known redundancy of HOX family in skeletal tissues there is a distinct pattern of HOX expression. Collectively, these results emphasize the importance of HOXB7 during in vivo skeletal formation and in postnatal skeletal homeostasis.

\section{DiscusSION}

Adult stem cells, as somatic cells, undergo an age-dependent decline in their number and function resulting in tissue aging and disease [43-45]. In addition, since their ex vivo isolation is often a prerequisite for regenerative medicine applications, 

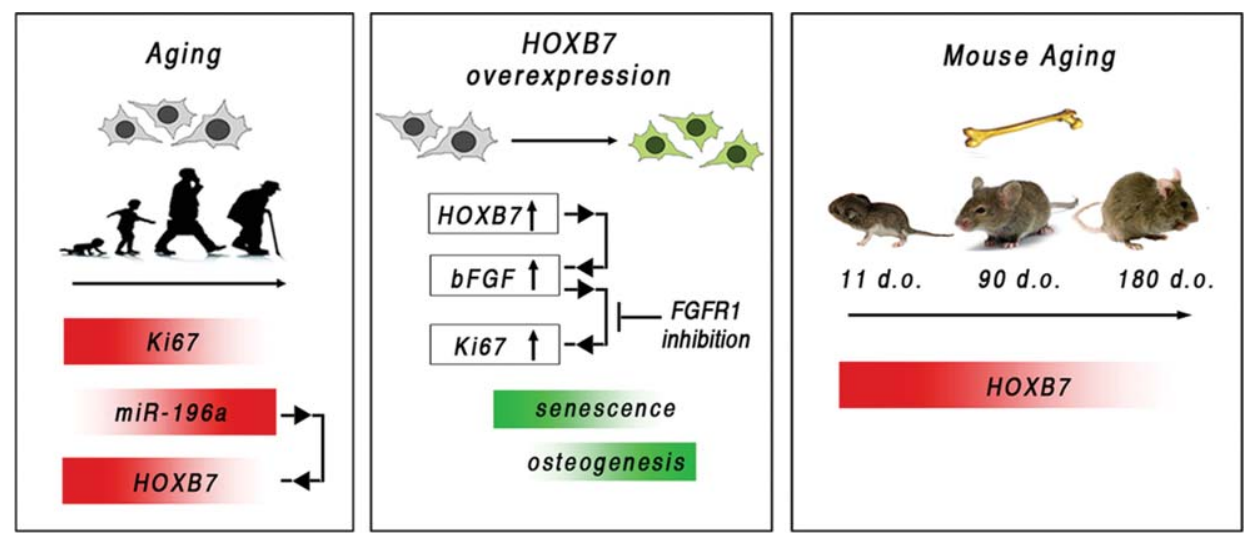

Figure 7. Summarizing diagram of HOXB7 impact on aging and mesenchymal stromal/stem cells (MSC) performance. (Left panel: aging) Unmodified human MSC show decreased levels of Ki67 during aging associated with an increase of miR-196a level and with a decrease of HOXB7 expression. (Middle panel: HOXB7 overexpression) Forced HOXB7 expression determines an increase of autocrine bFGF secretion, resulting in higher Ki67 expression, a reduction of senescence, and an improved osteogenesis. (Right panel: mouse aging) Skeletal aging in mice is associated with a progressive decrease of HOXB7 levels. Abbreviation: bFGF, basic fibroblast growth factor.

these impairments may limit stem cells performance in vitro and after transplantation [46].

In this study, we began considering age as a highly discriminating parameter demonstrating that MSC isolated from young donors have a substantial difference in proliferative attitude, confirming previous findings on the negative correlation between donor age and cell growth [5, 7-10].

Several investigators, in order to identify fingerprints in aged MSC, have explored chromatin remodeling, genomic integrity, and regulation of transcription focusing on telomerase activity [15, 47], DNA methylation [12], mRNA, and miRNA expression $[11,13,15,16]$. This pioneering research provided early insights on age-modulated genomic features contributing to the perception that aging represents a controlled developmental program. However, these observations were incapable to clarify specific player/s driving the age-related cell performance.

Here, we explored miRNA expression profile and, comparing the two highly age-distinct groups, we identified only seven differentially expressed miRNA on a platform of 723 (0.9\%). Among those, we focused on miR-196a whose levels negatively correlate with Ki67 expression, allowing to hypothesize a link between the proliferation potential of MSC during aging and the identified miRNA (Fig. 7). Decrease in cellular proliferation is a hallmark of aging contributing to a reduced tissue renewal and regeneration [11, 44]. Thus, we thought that miR-196a may represent a pivotal mechanism by which MSC progressively lose their properties. In a previous study on gene modified adipose progenitors, it has been suggested that a forced miR-196a overexpression has a negative influence on both proliferation and differentiation through HOX gene modulation [35]. Our data confirm these early findings, further indicating that miR-196a may also impact on proliferation in unmodified primary MSC and suggesting that miRNA-196a upregulation naturally occurs during aging at the progenitor level as previously suggested [48].

It is known that miR-196a has a critical role in the development regulating HOX expression [34]. The HOX family plays essential roles in limb morphogenesis and skeletal formation being also closely involved in stem cell proliferation and differentiation [49, 50]. Interestingly, the modulation of HOX expression has been previously associated with aging [12] and, in particular, HOXA5, HOXB3, and HOXB7 were repressed in aged MSC $[14,16]$.

For these reasons, we explored the connection between miR-196a, HOXB7, and MSC aging. The observed lower HOXB7 levels in adult group confirm the negative modulation of HOXB7 in aged cells and the original identification of HOXB7, as a target of miR-196a on primary human stem cell, suggests a miR-196a impact on cellular aging through HOXB7 expression.

Based on these relevant observations, we hypothesized that the higher proliferative potential of young MSC could be attributable to HOXB7 action. Thus, we forced HOXB7 expression obtaining a MSC cellular reprogramming in vitro associated with smaller size, increased levels of Ki67, and decreased $\beta$-gal positive cells with maintenance of immunophenotypic profile and no evidence of cytogenetic abnormalities. Previously, HOX have been studied in other human stem cells providing clear evidences of their regulatory functions and allowing to develop therapeutic strategies to optimize selfrenewal and commitment [23, 51]. For instance, HOXB4 overexpression enhances hematopoietic progenitors ex vivo expansion promoting their in vivo regenerative potential by multiple transcriptional activities leading to a modulation of several target genes $[52,53]$. Our data suggest that HOXB7 may represent the HOXB4 counterpart in MSC.

HOXB7 regulates several genes playing a significant role in cell proliferation, differentiation, and receptor signaling [27, 54-58]. In our model, we focused on bFGF, a known HOXB7 target [56] strongly involved in progenitor self-renewal, proliferation, and differentiation [59, 60]. HOXB7 modified MSC showed enhanced bFGF expression that may explain the induced MSC reprogramming, rendering bFGF a key factor to counteract aging. Additionally, using computational predictive algorithms (not shown), we were able to identify the FGFR3 as predicted target of miR-99a and miR-100, both found upregulated in aged cells, empowering the role of FGF in MSC aging and suggesting the need of deeper studies to reveal the epigenetic network ruling aging through FGF signaling.

In skeletal tissue, FGFs and their receptors maintain a sufficient pool of progenitors during bone development, growth, homeostasis, and repair in mammalians lifespan [59]. It has also been demonstrated that bFGF expanded MSC displayed in vitro a 
higher osteogenic differentiation, underlining the supporting role of bFGF in bone precursors [61]. Our data strongly follow these observations additionally indicating that MSC-HOXB7 have higher levels of secreted bFGF linked with a greater osteogenesis. Several evidences indicate that HOX are involved in skeletal development being also reactivated during fracture repair [21, 22, 41, 62]. Translating our findings during murine aging, we originally showed in postnatal life skeletal compartment, a progressively reduction of HOXB7 expression, suggesting that a similar dynamic may be relevant for humans (Fig. 7).

\section{Conclusions}

In conclusion, this research starting from an age-related comparison of a know stem cell type provides insights on the specific role played by miRNA in aged cells and identifies HOXB7 as a key factor to enhance cell performance and skeletal regeneration in ex vivo reprogramming strategies by bFGF secretion. The identification of HOXB7 decrease during aging in skeletal tissue may also retain important implication on prevalent degenerative pathologies, such as osteoporosis, prompting more investigations to better clarify the role of HOX in the context of human aging.

\section{ACKNOWLEDGMENTS}

This work was supported in part by the Italian Ministry of Health “Bando Cellule Staminali 2008" (MD, PP), Regione Emilia Romagna (PP, MD), and the Associazione ASEOP (PP).

\section{AUTHOR CONTRIBUtions}

O.C.: conception and design, collection and assembly of data, data analysis and interpretation, and manuscript writing; C.S., A.M., G.G, E.V., and M.S.P.: collection and assembly of data and data analysis and interpretation; M.F. and M.N.: collection and assembly of data on microarrays; F.G.: collection and assembly of data on karyotype; F.B.: collection and assembly of data on cell culture; E.M.H., P.C., and P.P.: conception and design and manuscript writing; M.D.: conception and design, financial support, data analysis and interpretation, manuscript writing, and final approval of manuscript.

\section{Disclosure of Potential Conflicts of INTEREST}

The authors indicate no potential conflicts of interest.

\section{REFERENCES}

1 Caplan Al, Bruder SP. Mesenchymal stem cells: Building blocks for molecular medicine in the 21st century. Trends $\mathrm{Mo}$ Med 2001;7:259-264.

2 Dominici $M$, Marino $R$, Rasini $V$ et al. Donor cell-derived osteopoiesis originates from a self-renewing stem cell with a limited regenerative contribution after transplantation. Blood 2008;111:4386-4391.

3 Li Y, Lin F. Mesenchymal stem cells are injured by complement after their contact with serum. Blood 2012;120:3436-3443.

4 Zant G Van, Liang Y. Concise review: Hematopoietic stem cell aging, life span, and transplantation. Stem Cells Transl Med 2012; 1:651-657.

5 Stenderup K, Justesen J, Clausen C et al. Aging is associated with decreased maximal life span and accelerated senescence of bone marrow stromal cells. Bone 2003;33:919926.

6 Sethe S, Scutt A, Stolzing A. Aging of mesenchymal stem cells. Ageing Res Rev 2006;5:91-116.

7 Mareschi K, Ferrero I, Rustichelli D et al. Expansion of mesenchymal stem cells isolated from pediatric and adult donor bone marrow. J Cell Biochem 2006;97:744-754.

8 Zhou S, Greenberger JS, Epperly MW et al. Age-related intrinsic changes in human bone-marrow-derived mesenchymal stem cells and their differentiation to osteoblasts. Aging Cell 2008;7:335-343.

9 Wagner $\mathrm{W}$, Horn $\mathrm{P}$, Castoldi $\mathrm{M}$ et al. Replicative senescence of mesenchymal stem cells: A continuous and organized process. PLoS One 2008;3:e2213.

10 Zaim M, Karaman S, Cetin $G$ et al. Donor age and long-term culture affect differentiation and proliferation of human bone marrow mesenchymal stem cells. Ann Hematol 2012;91:1175-1186.

11 Alt EU, Senst C, Murthy SN et al. Aging alters tissue resident mesenchymal stem cell properties. Stem Cell Res 2012;8:215-225.

12 Bork S, Pfister S, Witt $\mathrm{H}$ et al. DNA methylation pattern changes upon long-term culture and aging of human mesenchymal stromal cells. Aging Cell 2010;9:54-63.

13 Pandey AC, Semon JA, Kaushal D et al. MicroRNA profiling reveals age-dependent differential expression of nuclear factor $\kappa \mathrm{B}$ and mitogen-activated protein kinase in adipose and bone marrow-derived human mesenchymal stem cells. Stem Cell Res Ther 2011;2:49.

14 Alves H, van Ginkel J, Groen N et al. A mesenchymal stromal cell gene signature for donor age. PLoS One 2012;7:e42908.

15 Siegel G, Kluba T, Hermanutz-Klein U et al. Phenotype, donor age and gender affect function of human bone marrowderived mesenchymal stromal cells. BMC Med 2013;11:146.

16 Wagner W, Bork S, Horn P et al. Aging and replicative senescence have related effects on human stem and progenitor cells. PLoS One 2009;4:e5846.

17 Lim LP, Lau NC, Garrett-Engele P et al. Microarray analysis shows that some microRNAs downregulate large numbers of target mRNAs. Nature 2005;433:769-773.

18 Hackl $M$, Brunner $S$, Fortschegger $K$ et al. miR-17, miR-19b, miR-20a, and miR106a are down-regulated in human aging. Aging Cell 2010;9:291-296.

19 Noren Hooten N, Abdelmohsen K, Gorospe $\mathrm{M}$ et al. microRNA expression patterns reveal differential expression of target genes with age. PLoS One 2010;5:e10724.

20 Zhang X, Azhar G, Wei JY. The expression of microRNA and microRNA clusters in the aging heart. PLoS One 2012;7:e34688.
21 Gersch RP, Lombardo F, McGovern SC et al. Reactivation of Hox gene expression during bone regeneration. J Orthop Res 2005;23:882-890.

22 Leucht P, Kim J-B, Amasha R et al. Embryonic origin and Hox status determine progenitor cell fate during adult bone regeneration. Dev Camb Engl 2008;135:2845-2854. 23 Carè $A$, Valtieri $M$, Mattia $G$ et al. Enforced expression of HOXB7 promotes hematopoietic stem cell proliferation and myeloid-restricted progenitor differentiation. Oncogene 1999;18:1993-2001.

24 Dominici M, Rasini $V$, Bussolari $R$ et al. Restoration and reversible expansion of the osteoblastic hematopoietic stem cell niche after marrow radioablation. Blood 2009;114: 2333-2343.

25 Grisendi G, Annerén C, Cafarelli $L$ et al. GMP-manufactured density gradient media for optimized mesenchymal stromal/stem cell isolation and expansion. Cytotherapy 2010; 12:466-477.

26 Veronesi E, Murgia A, Caselli A et al. Transportation conditions for prompt use of ex vivo expanded and freshly harvested clinical-grade bone marrow mesenchymal stromal/stem cells for bone regeneration. Tissue Eng Part C Methods 2014;20:239-251.

27 Chile T, Fortes MA, Corrêa-Giannella ML et al. HOXB7 mRNA is overexpressed in pancreatic ductal adenocarcinomas and its knockdown induces cell cycle arrest and apoptosis. BMC Cancer 2013;13:451.

28 Ferracin $M$, Pedriali $M$, Veronese $A$ et al. MicroRNA profiling for the identification of cancers with unknown primary tissue-of-origin. J Pathol 2011;225:43-53.

29 Schmittgen TD, Livak KJ. Analyzing realtime PCR data by the comparative C(T) method. Nat Protoc 2008;3:1101-1108.

30 Warrens AN, Jones MD, Lechler RI. Splicing by overlap extension by PCR using asym- 
metric amplification: An improved technique for the generation of hybrid proteins of immunological interest. Gene 1997;186:29-35.

31 Marx JC, Allay JA, Persons DA et al. High-efficiency transduction and long-term gene expression with a murine stem cell retroviral vector encoding the green fluorescent protein in human marrow stromal cells. Hum Gene Ther 1999;10:1163-1173.

32 Tarte K, Gaillard J, Lataillade J-J et al. Clinical-grade production of human mesenchymal stromal cells: Occurrence of aneuploidy without transformation. Blood 2010 115:1549-1553.

33 Hermann A, List $\mathrm{C}$, Habisch $\mathrm{H}-\mathrm{J}$ et al. Age-dependent neuroectodermal differentiation capacity of human mesenchymal stromal cells: Limitations for autologous cell replacement strategies. Cytotherapy 2010;12:17-30.

34 Chen $C$, Zhang $Y$, Zhang $L$ et al. MicroRNA-196: Critical roles and clinical applications in development and cancer. J Cell Mol Med 2011;15:14-23.

35 Kim YJ, Bae SW, Yu SS et al. miR-196a regulates proliferation and osteogenic differentiation in mesenchymal stem cells derived from human adipose tissue. J Bone Miner Res 2009;24:816-825.

36 Philippidou P, Dasen JS. Hox genes: Choreographers in neural development, architects of circuit organization. Neuron 2013;80:12-34.

37 Boulet AM, Capecchi MR. Multiple roles of Hoxa11 and Hoxd11 in the formation of the mammalian forelimb zeugopod. Dev Camb Engl 2004;131:299-309.

38 Caré A, Silvani A, Meccia E et al. HOXB7 constitutively activates basic fibroblast growth factor in melanomas. Mol Cell Biol 1996;16:4842-4851.

39 Panek RL, Lu GH, Klutchko SR et al. In vitro pharmacological characterization of PD 166285, a new nanomolar potent and broadly active protein tyrosine kinase inhibitor. J Pharmacol Exp Ther 1997;283:1433-1444.

40 Tasso R, Gaetani M, Molino E et al. The role of bFGF on the ability of MSC to activate endogenous regenerative mechanisms in an ectopic bone formation model. Biomaterials 2012;33:2086-2096.

41 Chen F, Greer J, Capecchi MR. Analysis of Hoxa7/Hoxb7 mutants suggests periodicity in the generation of the different sets of vertebrae. Mech Dev 1998;77:49-57.

42 Chen F, Capecchi MR. Targeted mutations in hoxa-9 and hoxb-9 reveal synergistic interactions. Dev Biol 1997;181:186-196.

43 Bidwell JP, Alvarez MB, Hood M Jr et al. Functional impairment of bone formation in the pathogenesis of osteoporosis: The bone marrow regenerative competence. Curr Osteoporos Rep 2013;11:117-125.

44 Bellantuono I, Aldahmash A, Kassem M. Aging of marrow stromal (skeletal) stem cells and their contribution to age-related bone loss Biochim Biophys Acta 2009;1792:364-370.

45 Raaijmakers MHGP, Mukherjee S, Guo S et al. Bone progenitor dysfunction induces myelodysplasia and secondary leukaemia. Nature 2010;464:852-857.

46 Stolzing A, Jones E, McGonagle $D$ et al. Age-related changes in human bone marrowderived mesenchymal stem cells: Consequences for cell therapies. Mech Ageing Dev 2008;129:163-173.

47 Serakinci N, Hoare SF, Kassem $M$ et al. Telomerase promoter reprogramming and interaction with general transcription factors in the human mesenchymal stem cell. Regen Med 2006;1:125-131.

48 Li J, Dong J, Zhang Z-H et al. miR-10a restores human mesenchymal stem cell differentiation by repressing KLF4. J Cell Physiol 2013;228:2324-2336.

49 Phinney DG, Gray AJ, Hill K et al. Murine mesenchymal and embryonic stem cells express a similar Hox gene profile. Biochem Biophys Res Commun 2005;338:1759-1765.

50 Argiropoulos B, Humphries RK. Hox genes in hematopoiesis and leukemogenesis. Oncogene 2007;26:6766-6776.

51 Mahdipour E, Mace KA. Hox transcription factor regulation of adult bone-marrowderived cell behaviour during tissue repair and regeneration. Expert Opin Biol Ther 2011;11:1079-1090.
52 Antonchuk J, Sauvageau G, Humphries RK. HOXB4-induced expansion of adult hematopoietic stem cells ex vivo. Cell 2002;109: 39-45.

53 Schiedlmeier B, Santos AC, Ribeiro A et al. HOXB4's road map to stem cell expansion. Proc Natl Acad Sci USA 2007;104:1695216957.

54 Liao W-T, Jiang D, Yuan J et al. HOXB7 as a prognostic factor and mediator of colorectal cancer progression. Clin Cancer Res 2011;17:3569-3578.

55 Nguyen Kovochich A, Arensman M, Lay AR et al. HOXB7 promotes invasion and predicts survival in pancreatic adenocarcinoma. Cancer 2013;119:529-539.

56 Wu X, Chen H, Parker B et al. HOXB7, a homeodomain protein, is overexpressed in breast cancer and confers epithelialmesenchymal transition. Cancer Res 2006;66: 9527-9534.

57 Braig S, Mueller DW, Rothhammer T et al. MicroRNA miR-196a is a central regulator of HOX-B7 and BMP4 expression in malignant melanoma. Cell Mol Life Sci 2010;67: 3535-3548.

58 How C, Hui ABY, Alajez NM et al. MicroRNA-196b regulates the homeobox B7vascular endothelial growth factor axis in cervical cancer. PLoS One 2013;8:e67846.

59 Coutu DL, Galipeau J. Roles of FGF signaling in stem cell self-renewal, senescence and aging. Aging 2011;3:920-933.

60 Gabbianelli M, Sargiacomo M, Pelosi E et al. "Pure" human hematopoietic progenitors: Permissive action of basic fibroblast growth factor. Science 1990;249: 1561-1564.

61 Martin I, Muraglia A, Campanile G et al. Fibroblast growth factor-2 supports ex vivo expansion and maintenance of osteogenic precursors from human bone marrow. Endocrinology 1997;138:4456-4462.

62 Fendri K, Patten SA, Kaufman GN et al. Microarray expression profiling identifies genes with altered expression in adolescent idiopathic scoliosis. Eur Spine J 2013;22:13001311. 